\title{
Efficient Task Allocation Based on Green Computing in Private Cloud
}

\author{
Neeraj single \\ Chandigarh Engineering College, Landran, 140307, Mohali, Punjab, India \\ neeraj208031@gmail.com
}

\begin{abstract}
Cloud computing is a rapidly emerging new paradigm for delivering computing as a service. There are many research issues in cloud computing. Resource allocation is one of the challenging tasks in cloud environment. The main aim of resource allocation to reduce the infrastructure cost associated with companies. The resources offered in the cloud are probably heterogeneous and extremely dynamic. Due to this dynamic access, load balancing of jobs required. Cloud computing resource allocation should be elastic and intelligent, based on application demand and user requirements [1].Green cloud computing is a trend which has become popular with the emergence of internet driven services in every field of life. It refers to the prospective environmental advantages that computer based internet services can guarantee to the environment, by processing huge amount of data and information from collective resources pool.load balancing in an efficient way so that the resource utilization can be maximized and the energy consumption of the data centre could be minimized that can further result in reducing global warming. We have concluded the parameters that should be analysed and improved that will result in reduction of global warming and will increase the profits of cloud provider and the client. Cloud computing resource allocation should be elastic and intelligent, based on application demand and user requirements. Green cloud computing is a trend which has become popular with the emergence of internet driven services in every field of life
\end{abstract}

Keywords: Cloud Computing, Architecture, Challenges, Cloud Platforms, Research Issues

\section{INTRODUCTION}

Cloud Computing is an emerging technology that can support a broad-spectrum of applications. It is a new computing paradigm, where a large pool of systems are connected in public or private networks, to provide dynamically scalable infrastructure for data, application and file storage. Applications such as web conferencing, e-mail and customer relationship management (CRM), all run in cloud. It offers online infrastructure, data storage, and application. Hence, Cloud computing can be classified as a new paradigm for the dynamic provisioning of computing services supported by state-of-the-art data centers that usually employ Virtual Machine (VM) technologies for consolidation and environment isolation purposes. Many computing service providers including Microsoft, Google, Yahoo, and IBM are rapidly deploying data centers in various locations around the world to deliver Cloud computing services. Cloud computing is a type of Internet-based computing that provides shared computer processing resources on demand. It provides high elasticity with accessibility on computer resources for extremely small price. The main anxiety for society and industry is to consider the requests towards public cloud. The appearance of cloud computing is headed to comprehensive results on the system groups and networks of IT industries. Various benefits will construct cloud computing smarter; but, it lacks security models. With emerging information technology region, cloud computing should be approached with awareness and proper concern to the compassion of raw data. A Development plan helps computing environment to be as secure as possible and also in agreement with organizational policies. Thus data privacy is also preserved. It ensures complete profit from IT use for organizations. The objective of security concern for an organization is a key feature for making decisions about leasing IT services, exactly, for making decisions on shifting institute data, request, and additional resources to a public cloud. To increase efficiency and reduce the price, security and confidentiality should consider from the early forecast phase at the beginning of the product development life cycle [2]
Levels of the pedigree group

1.1Cloud architecture: The components of cloud computing as shown in Figure 1 are loosely coupled. It is broadly divided into two parts as follows

Front End- It is the client part. The best example for this is web browser.

1.2Back End- It is cloud itself: It is an important challenge for the next decades. In PAAS and SAAS, practical problems like license Management issues need to be resolved and current research is also addressing question on inter-operability and federation of cloud platform Cloud computing is considered as of the cutting edge design of an IT undertaking.

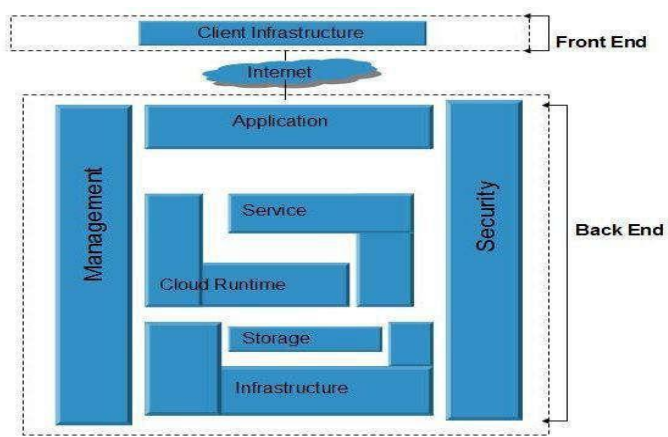

Fig 1. Cloud Architecture

\section{ESSENTIAL CHARACTERISTICS OF CLOUD COMPUTING}

Cloud administrations show five basic qualities that exhibit their connection to, and contrasts from, conventional figuring approaches.

On-request self-benefit: A shopper can individually arrange registering abilities as required and naturally, without human communication with a specialist organization. Figuring abilities are accessible over the system and get through standard instruments that advance by different customer stages.

Resource pooling: A supplier pool figures resources to serve a few customers utilizing a multi-occupant demonstrate which progressively allots and reassigns physical and virtual resources 


\section{GGCFIJCTR}

as indicated in a shopper request.

Fast flexibility: Capacities can be quickly and flexibly provisioned, by and large naturally, and quickly discharged to rapid scaling out and scaling in For a purchaser, the abilities have all the features of being boundless and can be acquired in any amount whenever.

Measured administration: Cloud frameworks control and upgrade resource utilization as a matter of course by utilizing a metering capacity as indicated by the kind of administration [3]. Utilization can be observed, controlled, and detailed, providing straightforwardness to both the supplier and the buyer.

\section{SEGMENTS OF CLOUD COMPUTING}

3.1Applications: Applications on request are called Software as a service (SAAS). Spending enormous amount for buying traditional software applications that insist on the functions needed for the operational business, one can pay for software that specially need for the business.

3.2Platforms: Platform as a service (PAAS) provides the facility of leasing the server space to run the software. This is typically a small amount to be spent for platform services. A buy server space can also be obtained through on demand service of the organization. The up gradation as well as down gradation of server space can also done as per the necessity.

3.3Infrastructure: Infrastructure as a service (IAAS) allows the users to lease the business tools such as Storage space, the tools that needed to develop their own applications. This also reduces the operational cost to the organization. The main concern of IAAS is resource optimization. This is viewed in this research view.

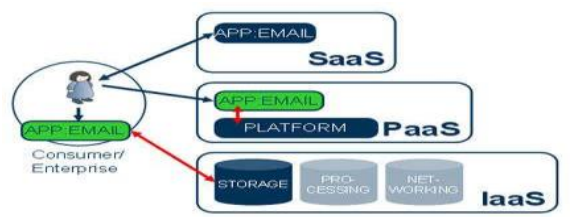

Fig 2 Segments of Cloud Computing

\section{BENEFITS OF CLOUD COMPUTING}

4.1Reduce Capital and Operations Costs: Investment of huge amounts for a venture or data center is not required. There is no need for dispenses with the need to prepare for provisioning. Permit organizations to begin little and increment their resources venture as and when required (pay-as-you-go).

4.2Simplify App Deployment and Management: Regular programming model is crosswise over portable, program, customer, server, cloud. Access to solid biological system of broadly sent applications is possible with reconciliation with existing IT resources (Software + Services).

\section{DEPLOYMENT MODELS}

5.1Public Cloud: Open cloud (off-webpage and remote) portrays cloud computing where resources are powerfully provisioned on an on-request, self-benefit premise over who charges onan utility processing premise.

5.2Private Cloud: A private cloud condition is often the initial step for an organization before embracing an open cloud activity [54]. Enterprises have found the advantages of combining shared administrations on virtualized equipment sent from an essential datacenter to serve nearby and remote clients.

5.3Hybrid Cloud: A half breed cloud condition comprises a bit of processing resources on location (on introduce) and off-site (open cloud). By coordinating open cloud administrations, clients can use cloud answers for specific capacities that are too expensive to keep up on-preface, for example, virtual server debacle recuperation, reinforcements and test/improvement situations.

\subsection{Community Cloud}

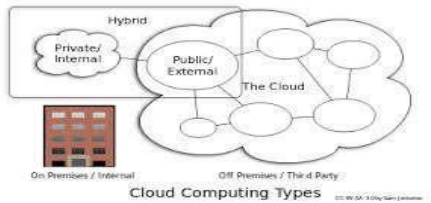

Fig 3. Cloud Computing Types

A people group cloud is framed when a few associations with comparable necessities share a basic foundation. Expenses are spread over a small number of companies clients open cloud to an more than a solitary inhabitant.

\section{VIRTUAL MACHINE}

A virtual machine (VM) is a product usage of a machine that executes programs very much like a physical machine. Virtual machines are isolated into two noteworthy characterizations, in the light of their utilization and level of correspondence to any genuine machine [57]. A framework virtual machine provides a total framework stage which bolsters the execution of an entire working framework (OS). These typically imitate a current design, and are worked with the reason for giving a stage to run programs where the genuine equipment is not accessible for utilize. A prepare virtual machine is intended to run a solitary program, which implies that it underpins as single process. Such virtual machines are generally well suited to at least one programming dialect and work with the motivation behind giving system convenience and adaptability. A fundamental normal for a virtual machine is that the product running inside is restricted to the resources and reflections gave by the virtual machine-it can't break out of its virtual condition.

6.1Data Centers: Engineers facilitate application in cloud to balance resources, while the expansion and diminishing their virtual frame work is done as per the request from customers. The programmers make this utilization additionally for their private use.

6.2 Resource allocation Resource administration incorporates resource disclosure, resource booking, resource allotment and resource checking. Resource revelation recognizes the appropriate physical resources in which the virtual machines are to be made coordinating the client's demand. Resource planning chooses the best resource from the coordinated physical resources.

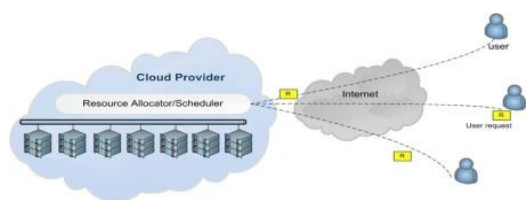

Fig 4. Resource allocations 
The primary target is to actualize resource distribution components that give a mechanized provisioning of resources. Along these lines, one of the fundamental motivations behind a few cloud administrators is to assign a timetable for the best usage in an accessible resource. An engineer's request faces, adjacent to product, a few data about the application's needs and administrations as arranged already. In this manner, the Cloud Computing administrator confronts the issue of choice of the most reasonable virtual resources and also the physical resources to suit for the application under the prerequisites arranged beforehand [4].

6.3Research Issues in Resource Allocation This sector describes the principle challenges confronted while managing Resource allocation under a Cloud. The difficulties that a RAS ought to face are isolated into four classifications, resources displaying and portrayal, resource offering and treatment, resource revelation and observing, and resource determination. When building up an resource allocation framework, one ought to consider how to portray the resources display in the Cloud. At the point when solicitations for resources arrive, the RAS ought to start resource disclosure to figure out whether there are to be sure resources accessible in the Cloud to go to the demand. At long last, if resources are accessible, the RAS may choose and apportion them to serve the demand. These difficulties are depicted with more subtle elements next.

6.4 Energy efficient in cloud environment Energy efficiency has emerged as a special problem in large data center and cloud providers. The datacenter include a large wide variety of web servers also called physical machines (PMs). The power consumption is that the key difficulty in content distribution machine and maximum disbursed systems (Cloud systems).

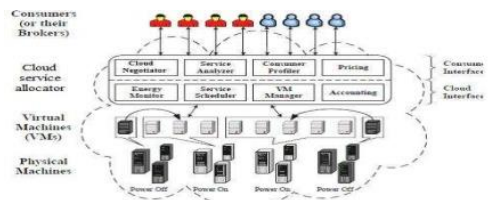

Fig 5. Energy efficient in cloud environment

Energy consumption is a key factor for cloud users as well as cloud providers. The problem in energy consumption can be divided into two parts. First one with server side operations another one is networking side communications. This research focused on VM migration, hybrid genetic algorithm to reduce the energy consumptions in data centers. This can be implemented in platform as a service segment. Schedulers are used to schedule the resources and the load balancers are used to balance the resources and to predict the load as well as to reduce the energy. To improve the energy efficient in cloud environments that should follows some methods.

6.5 Resource allocation algorithm Certain scheduling policies like Global scheduling policy employs the various details of the device to distribute work to the multiprocessor and also it regulates the performance of the system.

6.5.1 Static Scheduling Algorithms

The main advantage of this technique is both the resources of the grid and the complete work to be performed are scheduled in static scheduling. Each and every task that is to be performed is mapped to the resources and then it is left undisturbed or unchanged. As this unchanged, this scheduling algorithm to various applications are said to be static [6].

\subsubsection{Dynamic Scheduling Algorithms}

The principle behind dynamic scheduling algorithms is that the online based task allocation is performed for any particular application only based on the need and demand. The two major works that the Dynamic task scheduling includes, dynamic states of the system are to be valued and decision making.

\subsubsection{Heuristic Scheduling Algorithms}

Various answers to the problems that occur during scheduling are indicated using the heuristics. The main drawback of this system is most of the time it cannot give perfect solutions but consumes a good amount of cost. It also requires various resources of the system to perform their functions. Opportunistic Load Balancing. The methodology behind this balancing method is it assigns the entire task to the next system available as it does not take care of the execution time of that particular system, [5] and thus this method is considered to be simplest.

\section{LITERATURE SURVEY}

Many Resource optimizing algorithms have been implemented and tested for various problems in Cloud environment. Due to the diversity in nature of cloud resources, nature- inspired and heuristic-based algorithms are studied and implemented in literature. Any type of application involves scheduling for its good performance. In cloud environment, resource allocation is considered as NP-hard, and so many optimized algorithms have been proposed in literature. Scheduling refers to dynamic allocation of resources to the virtual machines, satisfying user requirements and application demands. Meta heuristics like Genetic algorithm, Particle swarm optimization, simulated annealing, Threshold accepting algorithm, Skewness algorithm, TRACON, etc, have been implemented for the Cloud resource management problem. These algorithms are designed and implemented to satisfy the user-defined objectives like Make span, Reliability, Completion time, Energy and constraints like Deadline and Budget.

Peng et. al (2018)has proposed an evaluation energy efficient virtual machine allocation and genetic based algorithm based meta heuristic which support a power aware VM request allocation of multiple sustainable cloud data centers.

Ronget. al (2018) has reviewed the progress of energy-saving technologies in high-performance computing, energy conservation technologies for computer room and renewable energy applications during the construction and operation of data centers. From multiple perspectives of energy consumption and environment protection,

Kian et. al (2018)has investigated the effectiveness of VM and host resource utilization predictions in the VM consolidation task using real workload traces. The experimental results show that the approach provides substantial improvement over other heuristic algorithms in reducing energy consumption, number of VM migrations and number of SLA violations.

Giacobbeet. al (2018)has applied a new strategy to reduce the carbon dioxide emissions in federated Cloud ecosystems. More specifically, they have discussed a solution that allows providers to determine the best green destination where virtual machines should be migrated in order to reduce the carbon dioxide emissions of the whole federated environment. 
Yakhchiet. al (2018)has presented an approach based on Cuckoo Optimization Algorithm (COA) to detect over-utilized hosts. They have employed The Minimum Migration Time (MMT) policy to migrate Virtual Machines (VMs) from the over-utilized hosts to the under-utilized hosts.

Qiuet. al (2018) has Proposed the functioning of CSB (Cloud Service Brokerage) as an intermediary between tenants and cloud providers that can bring about great benefits to the cloud market. CSBs buy the cloud resources, i.e., servers, with lower prices from cloud providers and sell the resources to the tenants with higher prices

Li et. al (2018) has proposed a novel Energy-aware Dynamic Task Scheduling (EDTS) algorithm based on DVS (Dynamic Voltage Scaling) to minimize the total energy consumption for smart phones, while satisfying stringent time constraints and the probability constraint for applications.

Xia et. al (2018) has presented a novel stochastic framework for energy efficiency and performance analysis of DVS-enabled cloud. This framework uses virtual machine request arrival rate, failure rate, repair rate, and service rate of datacenter servers as model inputs.

Nagpureet. al (2018) has proposed dynamic resource allocation system which allocated resources to cloud user. The skewness algorithm measure uneven utilization of multiple resources of each VMs and accordingly balances across VMs.

Yakhchiet. al (2017) has proposed a novel power aware load balancing method, named ICAMMT to manage power consumption in cloud computing data centers. They have exploited the Imperialism Competitive Algorithm (ICA) for detecting over utilized hosts

Chen et. al (2017) has proposed a cloud based framework to provide a customer-oriented energy management as a service (EMaaS) for green communities, which are formed as virtual retail electricity providers (REPs) by involved DERs (Distributed Energy Resources) providers.

Torraet. al (2017) has debated about the incentives that customers and data centers can adopt such measures and propose a new service type and pricing scheme that is economically attractive and technically realizable.

Kumar et. al (2017) has proposed a green cloud broker for resource procurement problem by considering the metrics of energy efficiency and environmental friendly operations of the cloud service provider. They have used mechanism design methods to decide the allocation and payment for the submitted job dynamically.

Chen et. al (2017) has proposed quantitatively analyzed the impact of server consolidation and temperature of cooling water on the total electricity and server maintenance costs in hybrid cooling data centers. To minimize the total costs,

Chen et. al (2017) has focused on "greening" demand response in multi-tenant data centers by designing a pricing mechanism through which the data center operator can efficiently extract load reductions from tenants during emergency periods for EDR(Emergency Demand Response).

Sivarasuet. al (2017)has described that the regional renewable resources can effectively be utilized for powering the MG (Micro Grid) to supply fixed and deferrable loads. Communication between residential consumers during energy transfer through MG and communication between MG to utility grid is also emphasized.

Wadhwa et. al (2017) has proposed a new technique to reduce the carbon emission and energy consumption in the distributed cloud datacenters having different energy sources and carbon footprint rates. They have used the carbon footprint rate of the datacenters in distributed cloud architecture

Reddy et. al (2016)has presented a system that handles real-time and non-real-time tasks in an energy efficient method without compromising much on neither reliability nor performance. Of the three processors, two processors i.e. the first and second, handle real-time tasks, using earliest-Deadline-First (EDF) and Earliest-Deadline-Late (EDL) scheduling algorithms respectively

Rocha et. al (2016) has stated a hybrid optimization model that allows a cloud service provider to establish virtual machine placement strategies for its data centers in such a way that energy efficiency and network quality of service are jointly optimized.

Roy et. al (2016)has proposed a green cloud enabled framework which envisioned the energy efficient way of a minimal discharge and rectification of the problem of high carbon production so as to increase the profit margin.

Chiang et. al (2016) has discussed an efficient green control (EGC) algorithm for solving constrained optimization problems and making costs/performances tradeoffs in systems with different power-saving policies. Simulation results show that the benefits of reducing operational costs and improving response times can be verified

Haqueet. al (2016) has proposes an approach for High Performance Computing cloud providers to offer such a Green SLA service. Specifically, each client job specifies a Green SLA, which is the minimum percentage of green energy that must be used to run the job.

Zakarya et. al (2016) hasdiscusses that organizations, industry, research laboratories and other academia to study and minimize the power requirements of digital and electronic devices especially the huge amount of computers in the global village.

Hukwuet. al (2016) has presents formulations and solutions for Green Cloud Environments (GCE) to minimize its energy consumption under new models by considering static and dynamic portions of cloud components, to reduce severe environmental impacts. To implement these objectives, an indepth knowledge of energy consumption patterns in cloud environment is necessary.

Javedet. al (2015) hasobserved that datacenters consumes a lot of power and emits a large amount of carbon dioxide that contributes largely to global warming. The rise in global warming has elevated the need for datacenters to adopt such techniques and technologies that can be helpful to overcome the negative impact on environment.

Lamb et. al (2015) hasdescribed power issues at data centers in South Africa based on the author's recent experience. The solutions to these power issues include virtualization of servers and data storage. Cloud computing has become the ultimate way to virtualized IT resources and to save energy

Yuan et. al (2015) has provides a comprehensive overview of the techniques and approaches in the fields of energy efficiency for data centers and large-scale multimedia services. They have highlights important challenges in designing and maintaining green data centers and identifies some of the opportunities in offering green streaming service in cloud computing frameworks.

Yamini et. al (2015) has focuses on reduction in energy 


\section{CGCDIJCTR}

consumption over the full equipment life cycle as the prime motivator for "green" application design; with energy reduction as the best measure of "greenness". Green IT refers to the study and practice of using computing resources in an efficient, effective and economic way.

Wuateret. al (2015) has proposed green computing in cloud computing due to its dynamic structure and property in online services, differs from current data centers in terms of power management

Yang et. al (2015) has proposed the problem of DCN planning. In particular, we have developed VPTCA as a novel DCN planning solution, incorporating a GA-based VM placement algorithm and a traffic configuration algorithm. VPTCA explicitly takes into account the relationship among the three main factors that impact operational expenses in DCs: (1) VM placement to maximize utilization and reduce computing cost, (2) traffic configuration to avoid the congestion and reduce transmitting delay, and (3) computing and transmitting load balance to increase DC utilization and reduce energy consumption. Based on results from our extensive simulations, VPTCA outperforms other two well-known traffic assignment algorithms in in providing more transmission capability with less energy consumption.

\section{RESOURCE ALLOCATION USING VMSHIFT ALGORITHM}

Cloud computing invites the user to balance the resource usage in runtime. Virtualization technology is used to allocate the resources of the datacenter in runtime with reference to the customer requirements and demands. Cloud computing is the virtual technology that permits the user to avail the resource as pay as go model. Third party service providers offer software platforms and infrastructure as per their needs and demands. Datacenters are the storage place that stores various resources that are needed for the cloud. Virtual machines are available in data centers. They take care of the resource allocation. The concept of resource allocation is to allocate the datacenter resources to the physical machine. This happens in the cloud environment. Cloud Computing is a common phrase for the submission of organized solutions over the internet. Cloud Computing enables organizations to manage sources as application rather than having to develop and maintain managing infrastructures in-house. Cloud Computing, assures several eye-catching benefits for companies and end clients. Three of the main benefits of cloud managing, include (1) Self alternatives and On-demand (2) Versatility (3) Pay per usage. (1) Cloud Computing is based on Self alternatives and Ondemand assistance design. The end client can move up managing sources for almost any kind of on-demand. The client should be allowed to get connected to the cloud for performing tasks like developing, applying, managing and organizing. The client should have the ability to access managing capabilities as and when they are required and without any relationship to the cloud assistance agency. If on a particular day the need improves several times, then the program should be versatile enough to meet up with that extra need, and should come back to the regular level when the need decreases. we have developed VPTCA as a novel DCN planning solution, incorporating a GAbased VM placement algorithm and a traffic configuration algorithm. VPTCA explicitly takes into account the relationship among the three main factors that impact operational expenses in DCs: (1) VM placement to maximize utilization and reduce computing cost, (2) traffic configuration to avoid the congestion and reduce transmitting delay, and (3) computing and transmitting load balance to increase DC utilization and reduce energy consumption. Based on results from our extensive simulations, VPTCA outperforms other two well-known traffic assignment algorithms in in providing more transmission capability with less energy consumption.

8.1 Resource allocation using VM shift algorithmCloud computing invites the user to balance the resource usage in runtime. Virtualization technology is used to allocate the resources of the datacenter in runtime with reference to the customer requirements and demands. Cloud computing is the virtual technology that permits the user to avail the resource as pay as go model. Third party service providers offer software platforms and infrastructure as per their needs and demands. Datacenters are the storage place that stores various resources that are needed for the cloud. Virtual machines are available in data centers. They take care of the resource allocation. The concept of resource allocation is to allocate the datacenter resources to the physical machine. This happens in the cloud environment. Cloud Computing is a common phrase for the submission of organized solutions over the internet. Cloud Computing enables organizations to manage sources as application rather than having to develop and maintain managing infrastructures in-house. Cloud Computing, assures several eyecatching benefits for companies and end clients. Three of the main benefits of cloud managing, include (1) Self alternatives and On-demand (2) Versatility (3) Pay per usage. (1) Cloud Computing is based on Self alternatives and On-demand assistance design. The end client can move up managing sources for almost any kind of on-demand. The client should be allowed to get connected to the cloud for performing tasks like developing, applying, managing and organizing. The client should have the ability to access managing capabilities as and when they are required and without any relationship to the cloud assistance agency. If on a particular day the need improves several times, then the program should be versatile enough to meet up with that extra need, and should come back to the regular level when the need decreases.

\subsection{Skewness Algorithm}

The existing algorithm, named Skewness algorithm, manages the workload on the server. The algorithm gives best performance only on Group of the server. In this new idea the virtual machines are moved without turning off the client server. Almost all the current services provide this facility making the movement of the virtual machine easier for the administrator.

Pseudo code for Skewness algorithm

$\mathrm{t}=0, \mathrm{r}=0$ rate $\lambda$ up to time $\mathrm{T}$;

Generate work $\mathrm{Wj}$;

$\mathrm{t}=\mathrm{t}+[-(1 / \lambda) \ln ($ work $\mathrm{Wj})]$. If $\mathrm{t}>\mathrm{T}$, then stop;

set $r=r 1$ and set $r=t$;

Place the work $\mathrm{Wj}$ in the queue in ascending order

Create a set of node $\mathrm{Na}$;

Label the node $\mathrm{Na}$

Assign the availability cost and response time to node $\mathrm{Na}, \mathrm{CA}$

$\infty, \mathrm{RT}_{\leftarrow \infty} \rightarrow$

if ( $\mathrm{Na} \neq$ empty) then

for each node $\mathrm{b}$ belongs to $\mathrm{Na}$, do 
CGCDIJCTR

calculate the expected finish time of the work $\mathrm{Wj}$

if the response time of the node $\mathrm{j}$ is less than the assigned response time, i.eRTj<RT, then

$\mathrm{RT}=\mathrm{RTj} ; \mathrm{x} \mathrm{j}$;

end for

else

for each node $b$ in the system do

calculate the availability cost of work $\mathrm{Wj}$ on node $\mathrm{b}, \mathrm{CAj}$

if the availability cost of the work on node $b$ is less than assigned availability cost, i.eCAj<CA then

$\mathrm{CA}=\mathrm{CAj} ; \mathrm{RT}=\mathrm{RTj} ; \mathrm{x} \mathrm{j}$;

end for

end if

22. WLmin=N1 ;LImin $=; / *$ Assume that node 1 is lightly loaded and its load capacity is $* 1$

for each node $\mathrm{b}$ belongs to $\mathrm{Na}$ do

calculate its work load LIb ;

if the load of the node $b$ is less than minimum load index, i.eLIb<LImin then

set the load index of $\mathrm{b}$ as the minimum load index LIb and node $\mathrm{b}$ is the lightly loaded node

Allocate work $\mathrm{Wj}$ to node $\mathrm{b}$

else

Allocate work $\mathrm{Wj}$ to node WLmin

end if

The main concept of the VM shift algorithm is to calculate the overload in the server. Here an intelligent interface is placed on the data center.

Hotspot Identification

Here a particular hotspot value is set as a threshold value to the CPU usage. It can be set as $90 \%$ and above, when a particular server's CPU usage is more than $90 \%$.

Cold spot Identification

It is a method for identifying the idle server. The threshold for the cold spot is set as $20 \%$ of the CPU usage.

Hot and Cold Spot Migration

This step starts with the sorting of hotspots in the descending order .It implies the hottest spot is first in the list and then cold.

\subsection{VM Shift Algorithms}

Input: Set of data items

Fix quasi-identifiers

Initiate VM shift algorithm

Set the number of virtual machines we need

Choose the load prediction randomly

For each load prediction calculate the hot spots

Find the idle machine

Calculate cold spots for load prediction.

End for

Initiate VM Migration

For each hot spot do the migration.

Find the IMPS

Repeat step 10 to 12 until we get Resource balance

End for

End VM Shift process

Check number of hot spots for each Virtual machine.

The process of VM shift algorithm is to checks the unevenness utilization of the server. This algorithm is placed on the Data center. If any unevenness occurred on the server, it provides the resource to the opposite server desires an equivalent resource and VM shifted to the opposite machine by reducing the overload. Thus, it will increase the performance of the server. The algorithm executes periodically to evaluate the resource allocation status based on the predicted future resource demands of VMs. It defines a server as a hot spot if the utilization of any of its resources is above a hot threshold. This indicates that the server is overloaded and hence some VMs running on it should be migrated away. The algorithm defines a server as a cold spot if the utilizations of all its resources are below a cold threshold. This indicates that the server is mostly idle and a potential candidate to turn off to save energy.

The algorithm sorts the list of hot spots in the system in descending Temperature. The goal is to eliminate all hot spots if possible. Otherwise, keep their temperature as low as possible.

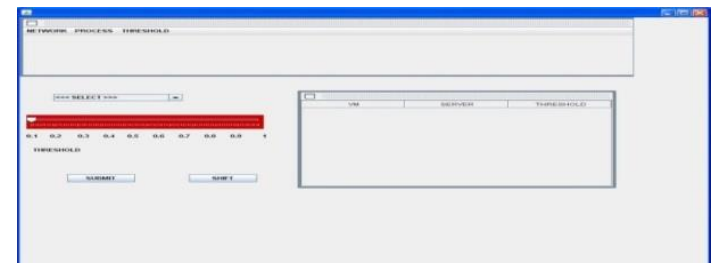

\subsection{Automated Resource Allocation}

When the research work focuses on the Resource Allocation, the Service level Agreement (SLA) should be satisfied. For this purpose, the Resource patterns are changed on the application and user demands, leading to the introduction of the concept called Auto Control. This manages all the different resources on the different users. Auto Control permits when the workload of the server changes; the image of the resource is also changed.

\subsection{Resource Allocation by Runtime}

Runtime migration is used for allocating resources. The main advantage of this system is that it satisfies the Service Level Agreement of users. Elimination of the overload with the physical machine has its beginning with the shifting of virtual machine to reduce overload. This result in increased performance of the overall systemonline migration begins with the identification of the volume of a physical machine and a virtual machine. The algorithm sorts out the physical and virtual machines on the basis of volume. Migration is done with the size of volume ration between the servers.

\subsection{Simulation Results}

The experimental result shows the number of hotspots with load prediction and without load prediction.VM wave simulation is the simulation tool that has been used for identifying the various Hotspots available. A graph has been plotted for hotspot identification for situations with and without load predictions. Hot Spot Identification: The number of virtual machine is taken on the $\mathrm{X}$-axis and number of hotspot on the $\mathrm{Y}$-axis. The algorithm sorts the list of hot spots in the system in descending Temperature. The goal is to eliminate all hot spots if possible. Otherwise, keep their temperature as low as possible.

In the graph (Fig. 8.6), the number of hot spots is less in load prediction. The reason for the above result is that the predicted load always gives best performance. Unpredicted load gives more hot spots due to the inability to get the exact load. 


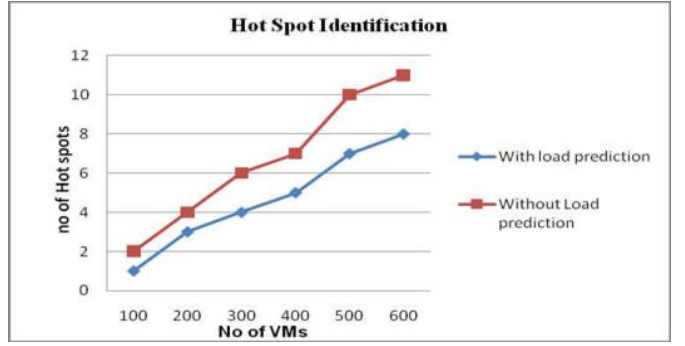

Fig 6. Number of hot spots

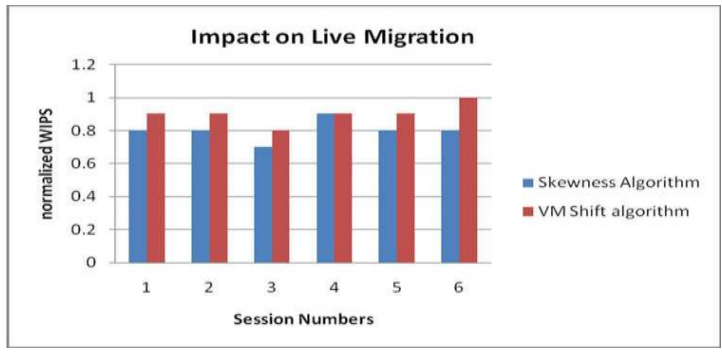

Fig 7. Hotspot and cold spot mitigation

When the VM broker is initialized, it displays the details of the VM broker. It also displays the starting and shutting down of the broker. It monitors the start time and completion time of VM. The final status of VM is considered as the output. Resource Balance

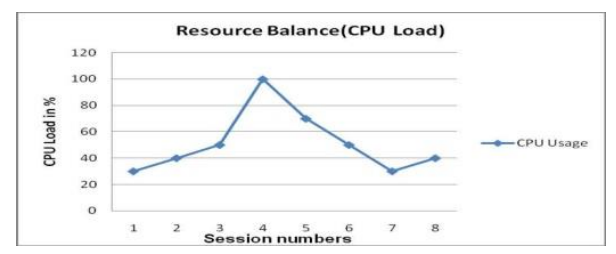

Fig 8. Resource balance in CPU load using VM Shift algorithm

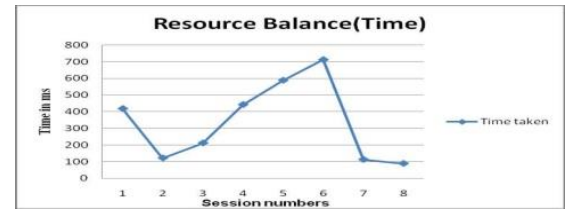

Fig 9. Resource balance in time using VM Shift algorithm

\subsection{Genetic algorithm based resource allocation}

Resource allocation in cloud computing should be flexible and need modification as per the availability of virtual machines in data center for allocation of the resources to the user. The algorithm for resource allocation should take decision based on dynamic information. In the existing work, an aggressive resource provisioning task using SPRNT algorithm [7] makes a dynamic allocation of the data center resources. The Existing work focuses on increased number of virtual machines and increased workload.The work should take a decision on the basis of dynamic information. This method lacks in memory access time.In general, cloud computing focuses on selfsolutions. These solutions are based on application demands. This means cloud environment ensures the flexibility pay per utilization model. Flexibility in cloud is implemented through handling and arranging the dynamic resources on a particular instance. When any extra needs an algorithm, it should consider the dynamic information. Cloud computing always falls in utilization price. There is no advanced price in it. In order to reduce the computational cost the algorithm should effectively manage the resources [8]. SPRNT system fails when the workload increases. This research work proposes an intelligent resource allocation based on artificial and computational intelligence. The algorithm sorts the list of hot spots in the system in descending Temperature. The goal is to eliminate all hot spots if possible. Otherwise, keep their temperature as low as possible

\subsection{Energy efficient resource allocation}

The emerging technology of cloud computing offers virtualization models and new computing models where resources such as online applications, computing power, storage and computing infrastructures can be shared as services through the web[1,4].The computing model adopted mainly by cloud computing providers (e.g., Microsoft, Google) is inspires features for customers whose demand on virtual resources varies with time $[1,8]$.The cloud provides suitable, on-demand service, elasticity, broad network access, resource pooling and measured service in highly customizable manner with minimal management effort $[3,8]$.Cloud programs are implemented in remote data centers (DCs) wherever high capability servers and storage systems are located. A quick growth of demand for cloud primarily based services results in an established order of large data centers taking high volume of electrical energy [7, 9]. Energy efficient model is needed for complete infrastructure for reducing functional prices, while maintaining very important Quality of Service (QOS). Energy improvement is achieved by way of combining resources as in keeping with the current usage, green virtual network topologies and thermal processing of computing hardware's and nodes[1,2].The objective of scheduling algorithms is to map tasks onto nodes and order their execution in an exceedingly thanks to optimize overall performance. In scheduling theory, the fundamental assumption is that each one machine is always available for the process. This assumption may well be affordable in some cases. However it is not valid in eventualities wherever there exist \& maintenance definite requirements, breakdowns, or different constraints, which make the machines unavailable for process [29]. Examples of such constraints are often found in several application areas. As an example, machine nodes in Heterogeneous systems in Cloud environment have to be sporadically

\subsection{Energy efficient in cloud environment}

Energy efficiency has emerged as a special problem in large data center \& and cloud providers. The datacenter include \& a large and wide variety of web servers, also called physical machines (PMs). The power consumption is that $\&$ in the key difficulty in content distribution machine and maximum disbursed systems (Cloud systems) [9]. These requirements are the buildup of networked computing resources from one or multiple companies on datacenters increasing over the world.

\subsection{Energy efficient algorithm}

As per earlier research the energy management has been applied in cloud datacenter [16]. This work, the arriving virtual machine (VM) makes a request to the cloud data center and provides exact physical machine (PM) to the cloud data center. This proposed work reduces energy consumption to the cloud Figure. 


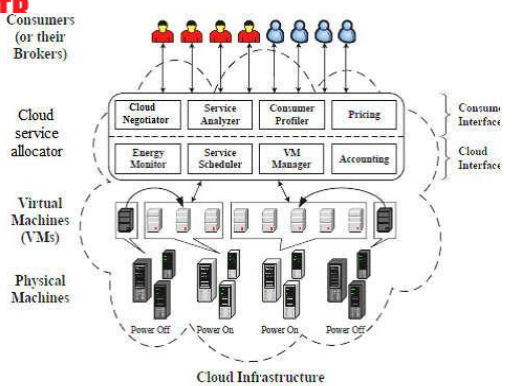

Fig 10. Resource balance in the cloud

\subsection{Energy efficient in cloud environment}

datacenter by sleep the unused PMs. This work proposes three frameworks, viz., data clustering, workload prediction and power management. These frameworks used analysis and observe the workload variations of the particular time. The data clustering method uses K-means the clustering algorithm. This algorithm groups any type of $\mathrm{VM}$ request.

Then power management method calculates the PM utilization using BFD algorithm. The proposed an energy aware resource allocation for scientific workflow execution in cloud environment. This method helps reducing the energy consumption of cloud platform using the energy aware resource allocation method. This method finish and analysis the idle virtual machines then migrates that virtual machine. Earlier research work focused on MADLVF algorithm for overcoming the problem in energy consumption and resource utilization of datacenter [10]This algorithm uses the VM consolidation method that reduces high energy consumption of datacenter. One of the most vital resources of cloud computing is the calculated or estimated processor. The components present in this processor are the transistor values ranging between thousands to billions. Processors with 2300 transistors having a cyclic frequency of about $740 \mathrm{KHz}$ were developed in the 19th century. In the current scenario, the clock frequency up to $3 \mathrm{GHz}$ can be achieved including transistors nearly 1.7 billion in number. A study suggests that in the rapid development continues, it is possible to develop a processor having transistors equal to the total neurons present in the human body by the year 2026. Loss

Reduction in the energy consumption of the distributed computing system can be achieved by means of using various algorithms or other techniques which leads to the improvement of the system performance. Some of the vital techniques such as Dynamic Voltage and Frequency scaling are used for reducing the energy in hardware level. Scheduling policies with Constraints in energy and estimation time are introduced for reducing the energy consumption in software level. It is evident from Arrhenius equation that heat generated from the system due to the increased level of energy consumption increases the overall system temperature which may lead to the breakdown of the various components for every $10 \mathrm{oC}$. In general, usage of energy in any processor increases with increase in the estimation time of the processor and vice versa.

An algorithm is designed for reduction in the energy consumption. In this algorithm, energy used by any processor is assumed to be the primary objective of the proposed algorithm which is as follows,

Step: 1 Position of the particle, its best fitness value and velocity are set randomly by considering the population.

Step: 2 Unit addition, comprehensive addition and inactivity assessment are initialized.

Step: 3 Primary objective functions are designed for reducing energy consumption.

Step: 4 Fitness value for each particle is calculated for a particular population.

Step: 5 Particle with low fitness value is selected and compared with its best fitness value.

Step: 6 The position of the particle and its speed are updated using mathematical Equations

\subsubsection{Fitness Function Generation}

In this approach the best virtual machines are selected by using the equation 5.1. The total fitness values are calculated using equation 5.2. Since hybrid genetic algorithm is a combination of a genetic algorithm, K-means and the migration algorithm, it is must to calculate the fitness value. This fitness value gives the best virtual machines in the data center. The cloudlet corresponding to virtual machines are selected for the migration process. CPU utilization between Energy aware algorithm, Heuristic algorithm and Hybrid genetic algorithm are explained. The number of cloudlets is taken in the $\mathrm{X}$ axis and number of virtual machines is taken in the $\mathrm{Y}$ axis. The initial parameter is set between 100 and 200 cloudlet. Number of cloudlet increases if number of virtual machines is increased. For example 100cloudlets are allotted with 10 virtual machines in the cases of Energy aware algorithm and Heuristic algorithm. But, for Hybrid genetic algorithm, 6 virtual machines are needed for the same number of cloudlet. This provides efficient CPU utilization on servers. So energy also reduced. In this approach the best virtual machines are selected by using the equation 5.1. The total fitness values are calculated using equation 5.2. Since hybrid genetic algorithm is a combination of a genetic algorithm, Kmeans and the migration algorithm, it is must to calculate the fitness value. This fitness value gives the best virtual machines in the data center. The cloudlet corresponding to virtual machines are selected for the migration process. Resource allocation starts in the next step. All the cloudlets are mapped with the physical machine available in the server side. The algorithm starts the migration process, when the clock frequently is increased. More energy consumed when the clock frequency increased. Cloud Simuse's Sim Java as the distinct occasion simulator engine that facilitates several primary features, such as lining up and handling of activities, development of cloud computing system organizations (services, variety, data middle, agent, VMs), interaction between elements, and control of the simulator time.

\section{CONCLUSION AND FUTURE WORK}

\subsection{Conclusion}

The proposed thesis overviews cloud computing and its background describing the architecture, models and benefits moving further towards the concept of green cloud computing as the energy efficiency is one of major problem with cloud computing. The proposed work thus puts forward an efficient energy consumption technique. Keeping in mind the problems formulated in the existing system. The proposed technique cloud environment is developed in java, deployed on cloud sim toolkit and the experimental results have been compiled as per quantitative analysis. In proposed techniques power saving in green cloud environment has been done using $\mathrm{k}$ means clustering at virtual machine level to classify the machines as low level and high level virtual machines. The cloudlets are also categorized 
into 3 parts: Suspend able cloudlets, cancellable cloudlets and non-permutable cloudlets.

\subsection{Future Work}

This work shows the energy consumption of the heterogeneous workload other processing element like no of CPU required by a cloudlet can also be considered to further increase the efficiency of work load consolidation techniques. To achieve green cloud computing server data center can use renewable energy resources like the solar system, bio gas plant energy.

\section{REFERENCES:}

[1]. Abishi Chowdhury and PriyankaTripathiEnhancing Cloud ComputingReliability Using Efficient Scheduling by Providing Reliability as a Service International Conference on Parallel, Distributed and Grid Computing, 14-20, 2014.

[2]. Abrishami S, Naghibzadeh $M$, and Epema D, Deadlineconstrained workflow scheduling algorithms for IaaS clouds, Future Generation Comput. Syst., 1400-1414, 2012.

[3]. Agarwal Y, Hodges S, Chandra R, Scott J, Bahl P, and Gupta R, Somniloquy:Augmenting Network Interfaces to Reduce Pc Energy Usage, Proc. USENIXSymp. Networked Systems Design and Implementation, 9-14, 2009.

[4]. Arindam Banerjee, Prateek Agrawal and Iyengar N. Ch. S. N Energy Efficiency Model for Cloud Computing International Journal of Energy,Information and Communications 29-42, 2013.

[5]. Armbrust M Above the Clouds: A Berkeley View of Cloud Computing, technical report, Univ. of California, Berkeley, 2225, 2009.

[6]. Azzedin F and Maheswaran M, Toward trust-aware resource management ingrid computing systems, In cluste computing and the grid,452-456,2002.

[7]. Bila N, Lara E D, Joshi K, Lagar-Cavilla H A, Hiltunen M, and Satyanarayanan M, Jettison: Efficient Idle Desktop Consolidation with PartialVM Migration, Proc. ACM European Conf. Computer Systems, 252-256, 2009.

[8]. Bobroff N, Kochut A, and Beaty K, Dynamic Placement of Virtual Machinesfor Managing SLA Violations, Proc. IFIP/IEEE Int'l Symp. IntegratedNetwork Management,22-27,2009.

[9]. Bodik P, Fox A, Franklin M J, Jordan M I, and Patterson D A, Characterizing,modeling, and generating workload spikes for stateful services, in Proc. 1stACM Symp. Cloud Computing, 241-252, 2014.

[10]. Bratton D and Kennedy J, Defining a Standard for Particle SwarmOptimization, in Swarm Intelligence Symposium, IEEE, 120-127, 2007. 